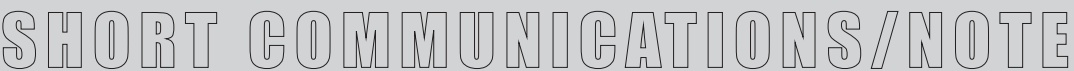

\section{I test di sensibilità ai farmaci antifungini (AST) in Italia: analisi di due indagini conoscitive a livello nazionale (I 999 e 2004)}

\author{
Claudio Farina ${ }^{1,7}$, Stefano Andreoni ${ }^{2,7}$, Gianluigi Lombardi ${ }^{3,7}$, Silvana Sanna ${ }^{4,7}$, \\ Esther Manso ${ }^{5,7}$, Paolo Fazii ${ }^{6,7}$, Gruppo di lavoro AMCLI sull'Antimicogramma ${ }^{8}$ \\ (Farina C., Andreoni A., Lombardi G., Sanna S., Manso E., Fazii P.)
}

'UO Microbiologia, AO "Ospedale San Carlo Borromeo", Milano

${ }^{2}$ Laboratorio di Microbiologia e Virologia, A.O. "Maggiore della Carità”, Novara

${ }^{3}$ Laboratorio di Microbiologia, Ospedale di Circolo e Fondazione Macchi, Università degli Studi dell'Insubria, Varese ${ }^{4}$ Dipartimento di Scienze Biomediche, Sezione di Microbiologia Sperimentale e Clinica, Università degli Studi, Sassari ${ }^{5}$ Laboratorio Analisi Chimico-Cliniche e Microbiologia, Azienda Ospedaliera Universitaria “Ospedali Riuniti”, Ancona 'Laboratorio Analisi Chimico-Cliniche e Microbiologia, P.O. "Spirito Santo", Pescara

${ }^{7}$ Comitato di Studio per la Micologia, Associazione Microbiologi Clinici Italiani

${ }^{8}$ vedi nota nei Ringraziamenti

Key words: Management, Antifungal susceptibility testing, Italy, Surveys, Laboratory

Antifungal susceptibility testing (AST) in Italy: management in two nation-wide surveys (I999 and 2004$)$

\section{SUMMARY}

The purpose of this study was to verify the standard procedures and minimum level of knowledge of Italian public laboratories involved in the management of antifungal susceptibility testing (AST). Two nation-wide surveys were performed in 1999 and 2004. 102 Italian hospitals located in 85 province capitals (82.5\%) participated to these surveys. In 1999, 28 (27.5\%) laboratories versus 16 (15.7\%) in 2004 stated that they did not perform any susceptibility testing. Some discrepancies observed in the survey confirm that AST is difficult to be correctly managed, and that it can be performed only in very well-trained centers. The great variability of the results of MIC determination and clinical interpretation underlines the urgent need to improve knowledge about indications, method choice and interpretative criteria for AST both for clinical microbiologists and clinicians.

\section{INTRODUZIONE}

I Laboratori di Microbiologia Clinica giocano un ruolo cruciale nella diagnosi rapida, completa ed accurata delle infezioni fungine. L'esecuzione dei test di sensibilità ai farmaci antifungini rappresenta un problema per il microbiologo, a causa delle sempre maggiori richieste da parte dei medici curanti e della mancanza di standardizzazione delle metodiche e dei criteri di interpretazione. Tuttavia, nel 2002 il Clinical Laboratory Standard Institute (CLSI) (8) e nel 2003 l'European Committe on Antimicrobial Susceptibility Testing (EUCAST) (4) hanno approvato un metodo di riferimento per la determinazione della sensibilità dei lieviti ai farmaci antifungini (AST) basato su un metodo di diluizione in brodo.

Lo scopo di queste indagini conoscitive, promosse dal Comitato di Studio per la Micologia (CoSM) dell'Associazione Microbiologi Clinici Italiani (AMCLI), è stato quello di analizzare le tecniche in uso presso i Laboratori di Microbiologia Clinica degli ospedali pubblici in Italia.

\section{MATERIALI E METODI}

Nel 1999 e nel 2004, 180 questionari sono stati inviati ai Direttori dei Laboratori di Microbiologia di tutti gli ospedali pubblici situati in 103 città in tutta Italia.

Il questionario era diviso in due sezioni:

1. Notizie generali:

a. Tipo di Laboratorio

b. Dimensioni dell'ospedale (N. di letti)

2. Informazioni tecniche:

a. Quando viene eseguito il test

b. Metodo in uso

c. Farmaci testati

d. Farmaci refertati

e. Criteri interpretativi adottati

f. Protocolli del Laboratorio

Ai Laboratori è stato chiesto di indicare anche:

1. Tipo di terreno utilizzato per $\mathrm{i}$ test di diffusione in agar

2. Tipo di sistema commerciale utilizzato per la determinazione delle MIC

3. Quali farmaci antifungini vengono saggiati 


\section{RISULTATI}

Curiosamente, in entrambe le indagini è stato restituito lo stesso numero di questionari $(102 / 180,56.7 \%)$. In particolare, sia nel 1999 che nel 2004 i questionari pervenuti provenivano da ospedali pubblici situati in 85/103 capoluoghi di provincia $(82.5 \%)$, anche se la provenienza geografica presentava alcune differenze tra le due indagini.

I risultati dei quesiti sono brevemente riassunti nelle seguenti sezioni con particolare attenzione all'argomento principale dello studio.

\section{Informazioni generali}

Allo scopo di analizzare i risultati, i Laboratori sono stati classificati in base al numero di letti dell'ospedale di appartenenza.

Lo studio del 1999 ha evidenziato che 8 ospedali su $102(7.8 \%)$ possedevano meno di 200 letti; 14 $(13.7 \%)$ avevano un numero di letti tra 201 e 400; $36(35.3 \%)$ tra 401 e 700 letti e $44(43.1 \%)$ più di 700 letti.

Nello studio del 2004, cinque ospedali su 102 (4.9\%) avevano meno di 200 letti, 14 (13.7\%) avevano un numero di letti tra 201 e 400 , e 47 $(46.0 \%)$ tra 401 e 700 letti e $36(35.3 \%)$ più di 700 letti.

Nel 1999, 64 laboratori (62.7\%) erano Servizi indipendenti oppure Istituti di Microbiologia, mentre 38 (37.3\%) erano Sezioni di Laboratori Clinici di grandi dimensioni, soprattutto negli ospedali di piccole e medie dimensioni situati in piccole città capoluogo di provincia. Invece, nel 2004 solo 26 Laboratori (25.5\%) erano autonomi, mentre $86(84.5 \%)$ erano Sezioni di Laboratori Clinici.

\section{Determinazione della sensibilità ai farmaci antifungini}

Nel 1999, 28 laboratori (27.5\%) contro i 16 $(15.7 \%)$ del 2004 affermavano di non eseguire alcun test di sensibilità ai farmaci antifungini. Per contro, 17 laboratori 16.7\%) nel 1999 contro i 13 $(12.7 \%)$ del 2004 riferivano che tale test faceva parte della loro routine.

In 57 ospedali (55.9\%) contro 73 (71.6\%) (rispettivamente nel 1999 e nel 2004) l'AST veniva eseguita solo su stipiti isolati da casi clinici selezionati. Nella tabella 2 é riportato chi decide se eseguire o meno il test: se il clinico o il microbiologo. Nella tabella 3 sono riassunti i criteri utilizzati per decidere quando eseguire l'AST e quale tipo di campioni clinici sono considerati idonei in caso di coltura positiva.

I metodi in uso sono riassunti nella tabella 4, confrontando i risultati dell'indagine del 1999 con quelli del 2004: il test di diffusione in agar era uti- lizzato rispettivamente in 6 laboratori $(8.1 \%)$ contro 1 solo $(1.2 \%)$; sistemi manuali per la determinazione delle MIC erano adottati da 58 laboratori (78.4\%) contro 82 (95.3\%). I metodi commerciali in uso sono elencati nella tabella 5. Le Concentrazioni Minime Inibenti (MIC) venivano interpretate in base ai criteri stabiliti nel Documento M27-A2 del Clinical Laboratory Standard Institute (CLSI). Undici laboratori $(10.8 \%)$ contro $3(3.6 \%)$ usavano contemporaneamente tecniche diagnostiche differenti.

I farmaci testati erano di solito quelli contenuti nei kit commerciali in uso.

Sessanta laboratori su $74(81.1 \%)$ contro $70 / 86$ $(81.4 \%)$ affermavano di refertare al medico curante tutti i farmaci saggiati (rispettivamente nel 1999 e nel 2004), mentre 5 laboratori (6.7\%) contro $6(7.0 \%)$ consideravano i risultati del test AST di esclusiva pertinenza del laboratorio e non li trasmettevano al clinico. Tuttavia, 9 laboratori $(12.2 \%)$ contro $10(13.7 \%)$ (rispettivamente nel 1999 e nel 2004) repertavano al medico curante solo i risultati di alcuni farmaci selezionati, quali Amfotericin B, 5-Fluorocitosina, Fluconazolo, Itraconazolo e Ketoconazolo.

\section{DISCUSSIONE}

In passato le segnalazioni di infezioni fungine erano poco frequenti sia nella letteratura scientifica italiana sia in quella internazionale. Nel corso degli ultimi 20 anni le micosi sistemiche sono state descritte con frequenza sempre maggiore, spesso connotate da notevole severità e da difficoltà diagnostiche e terapeutiche, soprattutto nei pazienti immunocompromessi (3).

La recente disponibilità di nuovi farmaci antimicotici, tuttavia, ha migliorato la percentuale di guarigione da queste malattie. Oggi è possibile gestire meglio il paziente rispetto al passato, a patto che il laboratorio sia in grado di pervenire ad un'identificazione accurata e che il medico

Tabella I. Dimensioni degli ospedali

\begin{tabular}{lrrrr}
\hline $\mathbf{N}^{\circ}$ LETTI & $\mathbf{1 9 9 9}$ & $\mathbf{2 0 0 4}$ & & \\
\hline$\leq 200$ & 8 & $(7.8 \%)$ & 5 & $(4.9 \%)$ \\
\hline $20 I-400$ & 14 & $(13.7 \%)$ & 14 & $(13.7 \%)$ \\
\hline $40 I-700$ & 36 & $(35.3 \%)$ & 47 & $(46.0 \%)$ \\
\hline$>700$ & 44 & $(43.1 \%)$ & 36 & $(35.3 \%)$ \\
\hline total & 102 & 102 & & \\
\hline
\end{tabular}

Tabella 2. Criteri decisionali adottati per l'esecuzione dei test AST

\begin{tabular}{lcccc}
\hline & 1999 & \multicolumn{2}{c}{2004} & \\
\hline RICHIESTA & & & & \\
DEL MEDICO & $27 / 57$ & $(47.4 \%)$ & $29 / 73$ & $(39.7 \%)$ \\
\hline DECISIONE DEL & & & & \\
MICROBIOLOGO & $30 / 57$ & $(52.6 \%)$ & $44 / 73$ & $(60.3 \%)$ \\
\hline
\end{tabular}


Tabella 3. Criteri microbiologici adottati per l'esecuzione dei test AST (indagine 2004)

\begin{tabular}{lccc}
\hline CAMPIONE & ESEGUITO DI ROUTINE & MAI ESEGUITO & ESEGUITO SU RICHIESTA DEL MEDICO \\
\hline Sangue & 74 & 4 & 8 \\
\hline Liquor cefalorachidiano & 71 & 7 & 8 \\
\hline Feci & 3 & 68 & 15 \\
\hline Urine & 3 & 26 & 57 \\
\hline Tampone faringeo & 10 & 54 & 22 \\
\hline Tampoe nasale & 8 & 57 & 21 \\
\hline Espettorato & 18 & 42 & 26 \\
\hline Fluidi da cavità sterili & 32 & 28 & 26 \\
\hline Essudato vaginale & 11 & 39 & 17 \\
\hline Essudato uretrale & 14 & 54 & 18 \\
\hline Cute ed annessi cutanei & 14 & 55 & 17
\end{tabular}

Tabella 4. Metodo utilizzato per $i$ test AST

\begin{tabular}{lcccc}
\hline & 999 & \multicolumn{3}{c}{2004} \\
\hline $\begin{array}{l}\text { I. DIFFUSIONE } \\
\text { IN AGAR }\end{array}$ & 6 & $(8.1 \%)$ & $1 / 86$ & $(1.2 \%)$ \\
\hline $\begin{array}{l}\text { 2. METODI } \\
\text { MANUALI }\end{array}$ & 58 & $(78.4 \%)$ & $82 / 86$ & $(95.3 \%)$ \\
\hline I. +2. & 10 & $(13.5 \%)$ & $3 / 86$ & $(3.6 \%)$ \\
\hline
\end{tabular}

Tabella 5. Metodi manuali utilizzati per i test AST (indagine 2004)

\begin{tabular}{lcc}
\hline Galleria ATB Fungus & 12 & $(15.2 \%)$ \\
\hline Strisce Etest & 8 & $(10.1 \%)$ \\
\hline Kit Candifast & 5 & $(6.3 \%)$ \\
\hline Pannello Sensititre & 38 & $(48.1 \%)$ \\
\hline E test + Sensititre & 5 & $(63 \%)$ \\
\hline ATB Fungus + Sensititre & 5 & $(6.3 \%)$ \\
\hline Kit Fungitest & 4 & $(5.1 \%)$ \\
\hline Galleria ATB Plus & 1 & $(1.3 \%)$ \\
\hline ATB Fungus +E Test & 1 & $(1.3 \%)$ \\
\hline totale & 79 &
\end{tabular}

curante sappia come utilizzare al meglio questi farmaci.

I test di determinazione della sensibilità ai farmaci antifungini vengono richiesti con maggiore frequenza da parte dei clinici; tuttavia, essi rappresentano tuttora un problema per il microbiologo, soprattutto a causa della mancanza di standardizzazione nei criteri di interpretazione.

Il notevole numero di laboratori che ha risposto al questionario indica un notevole interesse per la Micologia medica in tutta Italia. La distribuzione geografica dei laboratori partecipanti sul territorio nazionale era omogenea, cosicché è stato possibile ottenere un quadro attendibile della situazione italiana in un intervallo di 5 anni. Tuttavia, l'indagine ha interessato solo i laboratori degli ospedali pubblici, con la possibile esclusione di laboratori (anche specializzati) situati, ad esempio, in ospedali universitari.

La scelta di limitare 1'arruolamento ai soli laboratori degli ospedali pubblici situati in capoluoghi di provincia (pur in presenza del già citato bias) ha consentito di ottenere un quadro rappresentativo delle conoscenze micologiche sia da parte dei medici (che richiedono i test e che hanno in cura i pazienti affetti da micosi) sia da parte dei microbiologi (che eseguono gli esami e repertano $\mathrm{i}$ risultati).

Nel corso di un'indagine simile condotta nel 1992 in 94 laboratori situati in 73 capoluoghi di provincia il $18.1 \%$ dei partecipanti affermava di eseguire il test AST su tutti gli stipiti, mentre il $54.3 \%$ lo eseguiva solo in alcuni casi (5).

La tecnica di diffusione in agar era il metodo più comunemente utilizzato (30.9\%) nel 1992, sebbene occasionalmente il laboratorio non seguisse procedure standardizzate. Ciò era vero soprattutto per la scelta del terreno colturale, dato che molto spesso venivano utilizzati solamente Sabouraud Dextrose Agar oppure Agar Sangue. Anche i kit commerciali erano utilizzati frequentemente, anche se $\mathrm{i}$ metodi automatizzati non erano così comuni $(7.4 \%)$.

La maggior parte dei laboratori (76.1\%) refertava al medico tutti i farmaci saggiati, senza considerare il loro utilizzo clinico (sistemico o topico), la natura del micete isolato e del campioni clinico; 1'8.5\% dei laboratori non repertava alcun risultato ed il rimanente $15.4 \%$ refertava solo alcuni farmaci antifungini (principalmente 5-Fluorocitosina) e solo in casi specifici.

I risultati delle due indagini mostrano che la stessa percentuale $(27.5 \%)$ di partecipanti sia nel 1992 che nel 1999 riferiva di non eseguire alcun test AST, mentre questo numero scendeva al $15.7 \%$ nel 2004. La percentuale di laboratori che eseguivano AST su tutti gli isolati (16.7\%) o solo in alcuni casi (55.9\%) era simile nel 1992 e nel 1999. Tuttavia, l'analisi dei risultati dell'indagine del 2004 mostra che la percentuale di Laboratori che eseguono test AST era cambiata, probabilmente a causa di una migliore consapevolezza del problema da parte dei microbiologi. Infatti, la percentuale di laboratori che non esegue mai test AST era scesa al $12.7 \%$, mentre il $71.6 \%$ esegui- 
va AST in alcuni casi selezionati.

Come in precedenza, i farmaci saggiati in vitro erano quelli contenuti nei pannelli commerciali e quindi era impossibile scegliere $i$ farmaci da testare in base alle manifestazioni cliniche del paziente. I dati relativi ai criteri adottati per la refertazione dei risultati erano molto simili (sia nel 1999 che nel 2004) a quelli osservati nel 1992, indicando che a volte il microbiologo non dava al medico una risposta appropriata basata sull'utilizzo clinico del farmaco (sistemico o topico), sulla natura del fungo isolato e sul tipo di campione. Se i criteri di interpretazione erano spesso "personalizzati" (molti laboratori adottavano i criteri suggeriti dalla ditta produttrice invece di quelli raccomandati dal CLSI: 45.9\% nel 1992 contro 54.1\%, nel 1999), l'indagine del 2004 ha evidenziato che solo il $5.8 \%$ dei laboratori non segue i criteri dell'EUCAST o del CLSI $(4,8)$.

Anche se dall'inizio degli anni '90 non ci sono stati cambiamenti fondamentali nelle indicazioni e nell'interpretazione dei test $\mathrm{AST}$, in realtà sono stati introdotti notevoli miglioramenti tecnici. Rispetto ai risultati dell'indagine del 1992, nel 1999 è stata notata una maggiore attenzione nella scelta del terreno colturale (Mueller Hinton Agar e Sabouraud Dextrose Agar venivano utilizzati raramente), ma in realtà il test di diffusione agar veniva usato solo sporadicamente. I kit commerciali erano utilizzati molto spesso: i dati del 2004 confermano quelli ottenuti nel 1999 (rispettivamente $95.3 \%$ contro $78.4 \%$,), evidenziando l'andamento particolare del mercato italiano. Pochi Laboratori (10/74, 13.5\%) utilizzavano più di un metodo.

La situazione italiana è molto simile a quella rilevata negli Stati Uniti: il pannello Sensititre e le strisce Etest dominano il mercato $(64.5 \%)$, con una distribuzione delle due metodiche (usate da sole o contemporaneamente ad altre) in entrambi i Paesi. Infatti, il pannello Sensititre è il metodo più frequentemente utilizzato sia in Italia (60.7\%) sia negli USA (59\%), seguito dall'Etest (18.7\% contro 15\%) (7). La galleria ATB Fungus viene usata solo dal $15.2 \%$ dei laboratori italiani e solo 1 ' $1.2 \%$ utilizza il test di diffusione in agar. Invece, in Francia, i metodi più comunemente utilizzati sono l'ATB Fungus e l'Etest (rispettivamente $56 \%$ e $41 \%$ ) oppure una combinazione di 2 o più tecniche $(38 \%)$, mentre il 10\% dei laboratori esegue il test di diffusione in agar (1).

In conclusione, sembra che i laboratori italiani preferiscano metodiche più recenti quando devono adottare nuovi kit per i test AST, confermando sia l'interesse per i progressi in questo campo sia l'esistenza di un importante mercato. Alcune differenze osservate nel corso delle diverse indagini hanno confermato che $\mathrm{i}$ test AST sono difficili da gestire correttamente e che possono essere eseguiti solo in laboratori in cui opera personale ben addestrato. Inoltre, il basso livello di accuratezza nella refertazione suggerisce che la Micologia Medica dovrebbe essere insegnata sia come disciplina microbiologica sia come tecnica e che dovrebbe far parte del programma di insegnamento di Malattie Infettive. Questo problema è stato evidenziato dai risultati di numerose indagini nazionali in Italia ed in Francia $(2,6)$. La grande variabilità di risultati dei test di determinazione delle MIC e dell'interpretazione clinica sottolineano l'urgente necessità di migliorare le conoscenze riguardanti le indicazioni, la scelta dei metodi ed i criteri di interpretazione dei test AST sia da parte del microbiologo clinico sia da parte del medico curante. Infine, ci sembra utile raccomandare 1'esecuzione dei test AST solo in particolari casi clinici, anche se la standardizzazione delle nuove metodiche è nettamente migliorata rispetto al passato $(9,10)$.

\section{RINGRAZIAMENTI.}

Abruzzo- Chieti: Ospedale "SS Annunziata" (S. Martinotti; G. Mantini); "Ospedale San Camillo de' Lellis" (A. Procopio; A. Lattanzio); L'Aquila: Ospedale "San Salvatore" (S. Varrassi; G. Bruno); Pescara: Ospedale "Santo Spirito" (G. Riario Sforza; P. Fazii); Teramo: "Ospedale G. Mazzini” (G. Sciarpa, A. Fabrizi)

Basilicata- Potenza: AO "San Carlo" (A. Piperno; M. Mancino)

Calabria- Catanzaro: "Ospedale A. Pugliese" (M. Apollini; M. Masciari); Cosenza: "Ospedale Annunziata" (LM. Greco); Crotone: "Ospedale Civile San Giovani di Dio" (N. Di Maio; G. Ventura); Reggio Calabria: AO "BianchiMelacrino-Morelli" (G. Bolignano); Vibo Valentia: "Ospedale G. Sazzolino" (I.Vasapollo)

Campania- Avellino: Ospedale "San Giuseppe Moscati" (GP. Buonopane; A. Toschi); Caserta: AO "San Sabastiano" (A. Costanzo; I. Caserta); Napoli: Ospedale "D. Cotugno" (F. Romano; M. Conte); Ospedale "S. Giovanni Bosco" (R. deCapoa; G. Zorzato); Salerno AO "Ospedali Riuniti" (L. Avallone)

Emilia-Romagna- Bologna: AO "Ospedale S. Orsola-Malpighi” (MP. Landini; A. Nanetti); Cesena: Ospedale Civile (C. Delvecchio; P. Cipollini); Ferrara: AO "S. Anna" (F. Rigolin; MR. Rossi); Forli: "Ospedale MorgagniPierantoni” (G. Pallotti); Modena: Policlinico (F. Rumpianesi; C. Venturelli); Parma: Policlinico (C. Chezzi; L. Polonelli); Piacenza: AO "G. Da Saliceto" (E. Paggi, MM. Bertelli); Rimini: "Ospedale degli Infermi" (V. Ceroni; F. 
Castellani; R. Chicchi; A. Piscina); Ravenna: Ospedale "S. Maria delle Croci" (F. Benini, MF. Pedna); Reggio Emilia: AO "S. Maria Nuova" (L. Vecchia; G. Bertoldi)

Friuli-Venezia Giulia- Gorizia: Ospedale Civile (A. Troilo, F. Fontana); Pordenone: AO "S.Maria degli Angeli" (P. G. Mucignat; A. Camporese); Udine: Ospedale Santa Maria della Misericordia (E. Pitzus; P. Lanzafame)

Lazio- Latina: Ospedale "S. Maria Goretti" (C. Marchetti, L. Tega); Rieti: "Ospedale San Camillo de' Lellis" (C. Zepponi); Roma: Ospedale "L. Spallanzani" (E. Bordi; ML. deSantis); "Ospedale San Giacomo in Augusta" (L. Pacciani); Policlinico "Umberto $1^{\circ}$ " (M. Venditti); Viterbo: "Ospedale Grande degli Infermi" (R. Carrozza; I. Picari)

Liguria- Genova: Ospedale "San Martino" (OE. Varnier, O. Soro); Istituto "Gaslini" (R. Giacchino; ML. Belli); PO "San Carlo"- Voltri (L. Bottaio; O. Illiberi); Ospedale "Micone" (MC. Perrone; A. Bancheri); Imperia: "Ospedale Civile" (M. Bovina; AM. Vassallo); La Spezia: Ospedale "S. Andrea" (M. Dono); Savona: Ospedale "San Paolo" (F. Minetti; R. Bona)

Lombardia- Bergamo: AO "Ospedali Riuniti" (A. Goglio; A. Grigis); Brescia: AO "Spedali Civili" (N. Manca, G. Pinsi); "Ospedale S. Orsola Fatebenefratelli" (F. Pirali); Como: AO "Ospedale S. Anna" (G. Giana; M. Spinelli); "Ospedale Valduce" (R. Terramocci); Cremona: AO "Istituti Ospitalieri" (L. Ferrari); Lecco: AO "Ospedale A. Manzoni" (R. Vaiani); Lodi: "Ospedale Maggiore" (F. Pavesi; A. Ceraminiello); Milano: AO "Ospedale Maggiore" (A. Pagano; MA. Viviani); AO "Ospedale L. Sacco" (MR. Gismondo; C. Bonaccorso); AO "Ospedale Riguarda" (A. Astolfi); AO "Ospedale San Carlo Borromeo" (C. Farina; S. Perin); AO "Istituti Clinici di Perfezionamento" (E. Scarazatti; ML. Garlaschi); Ospedale "M. Melloni" (M. Saudelli, S. Lanzafame); "Ospedale San Giuseppe" (A. Archenti); "Ospedale Buzzi" (M. Facchini); "Ospedale San Raffaele" (M. Clementi; MC. Ossi); Pio Albergo Trivulzio (A. Berlusconi); Istituto Tumori (E. Clerici); Mantova: AO "Ospedale C. Poma" (F. Manzato; R. Ceruti); Pavia: Policlinico "San Matteo" (P. Marone); Sondrio: AO "Ospedale Civile" (L. Guerrini; C. Vitali); Varese: "Ospedale di Circolo-Fondazione Macchi" (A. Toniolo; GL. Lombardi);

Marche- Ancona: AO "Ospedali Riuniti" (M. Tocchini; E. Manso); Ascoli Piceno: "Ospedale Mazzoni" (P. Staffolani; A. Mariani); Macerata: "Ospedale Generale" (R. Cerolini; G. Orlandi); Pesaro: AO "Ospedale San Salvatore" (G. Rinaldi)
Molise- Campobasso: "Ospedale A. Cardarelli" (E. Ciotoli); Isernia: Ospedale "F. Veneziane" (P. Di Iorio; R. Ottaviano)

Piemonte- Alessandria: "Ospedale Santi Antonio e Biagio, C. Arrigo" (V. Carraccio); Asti: "Ospedale Civile" (AM. Fodale; G. Monticone); Biella: "Ospedale Degli Infermi" (P. De Toma; A. Marabaila); Cuneo: AO "Ospedale S. Croce" (M. Osenda); Novara: "Ospedale Magiore della Carità" (G. Fortina; S. Andreoni); Torino: ASO OIRM "S. Anna" (MA Latino; G De Intinsi); Ospedale Evangelico Valdese (M. Saitta, A. Sacchi); Verbania: "Ospedale San Biagio" (C. Perazzi; C. Rossi); Vercelli: Ospedale "S. Andrea" (GC. Guazzotti; F. Milano)

Puglia- Bari: Policlinico (S. Barbuti; MT. Montagna); Brindisi: Ospedale "A. Di Summa" (M. Scoditti); Foggia: AO "Ospedali Riuniti" (R. Antonetti; A. Di Taranto); Lecce: "Ospedale Vito Fazzi" (M. Pizzolante); Taranto: "Ospedale San Giuseppe Moscati" (A. Misserini; S. Fracchiolla) Sardegna- Cagliari: Ospedale "Binagli" (G. Satta; Podda); AO "Ospedale Brotzu" (A. Palomba; MG. Garau); "Ospedale SS Trinità" (AV. Carluccio; B. Saddi); "Ospedale Marino" (M. Sanna); Sassari: Dip. Scienze Biomediche (P. Cappuccinelli; S. Sanna); PO "SS Annunziata" (GB Cherchi, N. Castiglia); Oristano: "Ospedale San Martino" (A. Angius).

Sicilia- Agrigento: Ospedale "S. Giovanni di Dio" (F. Ferrara, E. Ghiraldi); Caltanissetta: AO "Ospedale S. Elia" (F. Santagati; A. Varvaro); Catania: "Ospedale Garibaldi- S.Luigi- Ascoli Tomacelli" (F. Divincenzo; D. Linà; A. Dinaso); Messina: Policlinico Universitario (G. Teti; Delfino); Ragusa: "Ospedale Civile" (C. Finocchiaro); Siracusa: Ospedale "Umberto $1^{\circ "}$ (C. Vaccarisi, L. Bozzanca); Trapani: Ospedale "S.A. Abate" (D'Angelo, Fedele)

Toscana- Arezzo: Ospedale "San Donato" (M. Badii; I. Galanti); Carrara: "Ospedale Civile" (P. Tuzi); Firenze: "Ospedale Careggi" (PL. Nicoletti; P. Pecile); Grosseto: "Ospedale della Misericordia" (F. Mancieri); Pisa: "Ospedale S. Chiara" (B. Innocenti; P. Leonetti); Livorno: Ospedale Civile (A. La Gioia); Lucca: "Ospedale Campo di Mare" (A. Savarino; R. Mattei); Pistoia: AO "Ospedali Riuniti” (R. Rossetti); Prato: Ospedale USL4 (G. Azzurri; P. Caprini; R. degl'Innocenti); Siena: Policlinico "Le Scotte" (GM Rossolini, E. Mantegoli)

Trentino-Alto Adige- Bolzano: Ospedale Civile (A. Lang); Trento: Ospedale "S. Chiara" (P. Caciagli; R. Sartori)

Umbria- Perugia: "Ospedale Monteluce" (M. Romagnoli; A. Repetti); "Ospedale R. Silvestrini" (F. Bistoni; D. Crotti); Terni: AO "Santa Maria" 
(P. Napoleoni; A. Colananni)

Valle d'Aosta- Aosta: "Ospedale Regionale della Valle d'Aosta" (P. Montanara; P. Lorenzetti)

Veneto- Belluno: Ospedale "San Martino" (G. Berciato; C. Zasio); Rovigo: Ospedale Civile (R. Pugina); Treviso: Ospedale "Ca' Fondello" (R. Rigoli); Venezia: Ospedale Civile (M. Gion); "Ospedale Umberto 1 " - Mestre' (F. Cantori); Verona: Ospedale "Maggiore" (R. Fontana; G. LoCascio; A. Zorzi); Vicenza: "Ospedale San Bortolo" (M. Rassu)

\section{BIBLIOGRAFIA}

1. Blanc V, Eloy O, Sanchez R, Mallié M, CoIBVH. Antifungal susceptibility testing in 62 French hospitals. J Mycol Méd 2005; 15: 197-210.

2. Eloy $\mathrm{O}$, Blanc V, Mallié $\mathrm{M}$, et al. Identification and antifungal susceptibility testing of two strains of Candida in 95 French hospitals. J Mycol Méd 2005; 15: $117-126$

3. Espinel-Ingroff A, Barchiesi F, Hazen KC, et al. Standardization of antifungal susceptibility testing and clinical relevance. Medical Mycology 1998; 36 (S1): 68-78.

4. European Committe on Antimicrobial Susceptibility testing. EUCAST discussion document E.Dis 7.1 June 2002. Method for the determination of minimum inhibitory concentration (MIC) by broth dilution of fermentative yeasts. Clin Microbiol Infect 2003; 9: 1-8.

5. Farina C, Picerno G, Medical Mycology Committee of AMCLI. Status of Medical Mycology in clinical laboratories in Italy. Alpe Adria Microbiology Journal 1995; 3: 197-209.

6. Farina C, Mazzoni A, Andreoni S, et al. Microbiologia Medica 2000; 15: 420-5.

7. Jones RN, Fritsche TR, CAP-MRC Members. Accuracy for antibacterial an antifungal susceptibility tests: report from the College of American Pathologists (CAP) Microbiology Proficiency Survey Program for 2004-2005. 45th Interscience Conference on Antimicrobial Agents and Chemotherapy, Washington DC, December 16-19, 2005, poster D$1654 / 75$.

8. National Committee for Clinical Laboratory Standards (NCCLS). Reference method for broth dilution antifungal susceptibility testing of yeasts. $2^{\text {nd }}$ ed. Approved Standard M27-A2. National Committee for Clinical Laboratory Standards, Wayne, Pa 2002.

9. Negroni R, Ellis D, Bulmer G, et al. Teaching medical mycology in the year 2000. Med Mycol 1998; 36 (S1): 106-8.

10. Reilly AA, Salkin IF, McGinnis MR, et al. Evaluation of Mycology Laboratories proficiency testing. J Clin Microbiol 1999; 37: 2297-2305.

\section{Claudio Farina}

UO Microbiologia

AO "Ospedale San Carlo Borromeo"

Via Pio II, 3 - 20153 Milano

Tel.: +39 02 40222456;

Fax: +3902 40222829

E-mail: farina.claudio@sancarlo.mi.it 